\title{
Electric Field Effects in the Presence of Chemi-Ionization on Droplet Burning
}

\author{
A. Patyal ${ }^{a}$, D. Kyritsis ${ }^{b}$, M. Matalon ${ }^{a}$ \\ ${ }^{a}$ University of Illinois at Urbana-Champaign, Urbana, IL 61801, USA \\ ${ }^{b}$ Khalifa University, Abu Dhabi, UAE
}

\begin{abstract}
The effects of an externally applied electric field on the burning characteristics of a spherically symmetric fuel drop, including the flame structure, flame standoff distance, mass burning rate and flame extinction characteristics of the diffusion flame are studied. A reduced three-step chemical kinetic mechanism that reflects the chemi-ionization process for general hydrocarbon fuels has been proposed to capture the production and destruction of ions inside the flame zone. Due to the imposed symmetry, the effect of the ionic wind is simply to modify the pressure field. Our study thus focuses exclusively on the effects of Ohmic heating and kinetic effects on the burning process. Two distinguished limits of weak and strong field are identified, highlighting the relative strength of the internal charge barrier compared to the externally applied field. For both limits, significantly different charged species distributions are observed. An increase in the mass burning rate is noticed with increasing the strength of the electric field in both limits, with a small change in flame temperature. Increasing external voltages pushes the flame away from the droplet and causes a strengthening of the flame with a reduction in the extinction Damkhöler number.

Keywords: chemi-ionization, electric field, Ohmic heating, ionic wind, droplet combustion, diffusion flame, flame extinction
\end{abstract}

\section{Nomenclature}

$B \quad$ Spalding Transfer number

$c_{p_{i}} \quad$ Specific heat at constant pressure of species ' $\mathrm{i}$ '

$\mathcal{D}_{i} \quad$ Abbreviate notation for the binary diffusivity $\mathcal{D}_{i N}$

$D_{i} \quad$ Scaled Damköhler number of reaction step ' $\mathrm{i}$ '

$\mathcal{D}_{i j} \quad$ Binary diffusion coefficient

$\mathcal{D}_{\text {th }} \quad$ Thermal diffusivity

$D a_{i} \quad$ Damköhler number of reaction step 'i'

$E_{i} \quad$ Activation energy of reaction step 'i'

E Electric field

$\mathbf{f}_{\mathbf{i}} \quad$ Electric body force per unit mass of species ' $\mathrm{i}$ '

g Gravitational acceleration

$h \quad$ Enthalpy of mixture

$h_{i} \quad$ Enthalpy of species ' $\mathrm{i}$ '

$h_{i}^{o} \quad$ Enthalpy of formation at $T^{o}$ for species ' $\mathrm{i}$ '

$k_{b} \quad$ Boltzmann constant

$L_{\mathrm{v}} \quad$ Latent heat of vaporization

$L e_{i} \quad$ Lewis number of species ' $\mathrm{i}$ '

$M \quad$ Mass burning rate $n_{i} \quad$ Number density of ionic species ' $\mathrm{i}$ '

$N \quad$ Total number of species in the system

$\mathcal{N}_{A} \quad$ Avogadro's constant

$p \quad$ Pressure

$q_{i} \quad$ Magnitude of charge on species ' $\mathrm{i}$ '

$Q_{i} \quad$ Heat of combustion of reaction step 'i'

$r \quad$ Radial distance from center of the droplet

$r_{o} \quad$ Initial droplet radius

$\mathcal{R} \quad$ Universal gas constant

$t \quad$ Time

$T \quad$ Temperature

v Bulk velocity of the gas mixture

$\mathbb{V} \quad$ Imposed voltage at droplet surface

$\mathbf{V}_{i} \quad$ Diffusion velocity of species ' $\mathrm{i}$ '

$\bar{W} \quad$ Mixture molecular weight

$W_{i} \quad$ Molecular weight of species ' $\mathrm{i}$ '

$X_{i} \quad$ Mole fraction of species ' $\mathrm{i}$ '

$Y_{i} \quad$ Mass fraction of species ' $\mathrm{i}$ '

Email addresses: patyal2@illinois.edu (A. Patyal ${ }^{a}$ ), dimitrios.kyritsis@kustar.ac.ae (D. Kyritsis ${ }^{b}$ ), mataloneillinois.edu (M. Matalon ${ }^{a}$ ) 


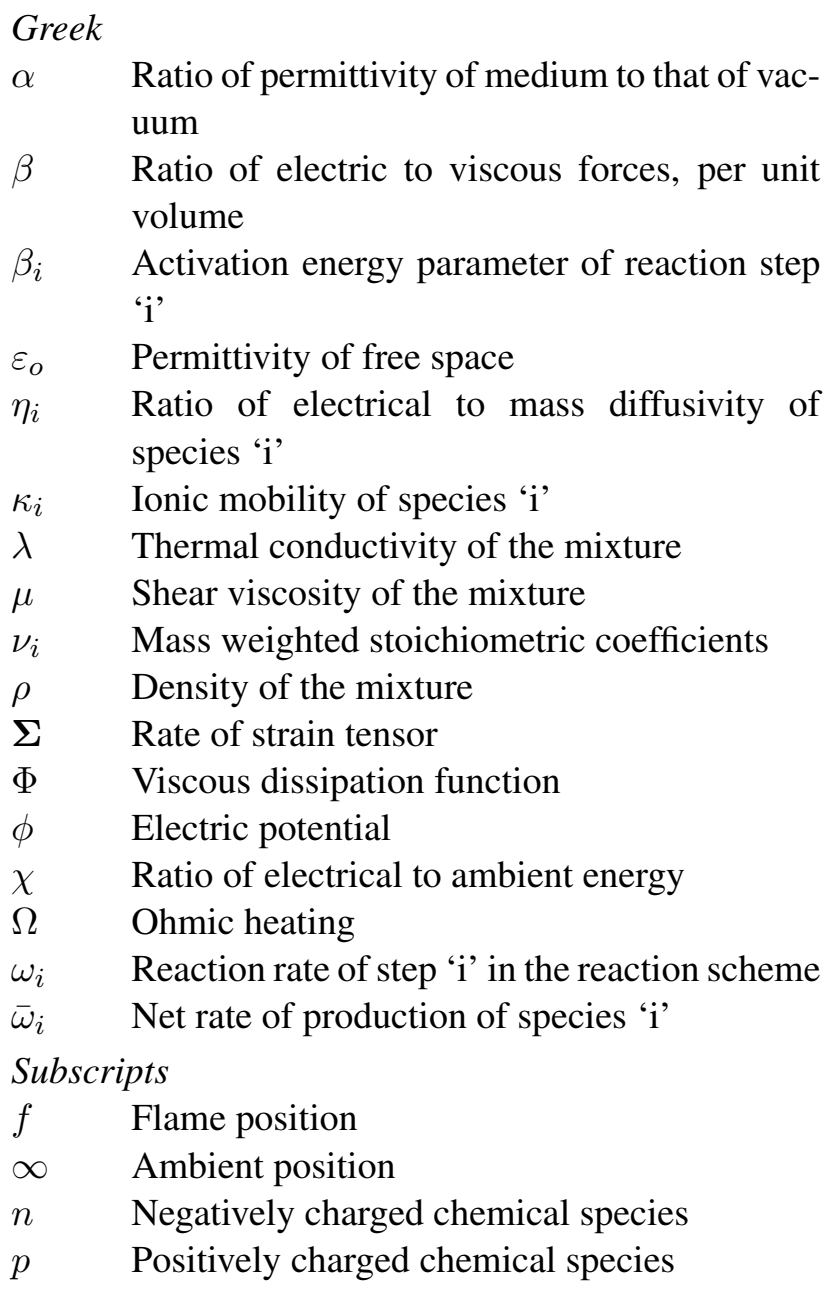

\section{Introduction}

Understanding the physics of combustion of nonpremixed reactants was greatly enhanced by the seminal work of Burke and Schumann [1] who introduced the infinitely fast chemistry limit. Since the chemical reaction occurs instantaneously as the fuel and oxidizer come in contact, a flame is established at the location where the separated reactants diffusing into each other meet, mix and react. The reactants are completely consumed at the flame, which separates a region where there is fuel but no oxidizer from a region where there is only oxidizer. Mathematically, this description corresponds to the asymptotic limit of an infinite Damköhler number, whereby the chemical reaction time is much shorter than the flow time. It was later recognized by Liñán [2] that the effects of finite rate chemistry can be taken into account by further assuming that the activation energy of the chemical reaction is large compared to the thermal energy of the cold reactants. Using an appropriately defined distinguished limit, he extended the flame description from complete combustion, corresponding to an infinite Damköhler num- ber, down to a large but finite Damköhler number associated with flame extinction. The effects of non-unity and distinct Lewis numbers, for equi-diffusion flames ${ }^{1}$, were later introduced by Cheatham and Matalon [3] who also provided expressions for the fuel and/or oxidizer leakage through the reaction zone as a function of the Damköhler number. This theoretical development was corroborated with numerical studies based on detailed chemistry and transport, that provided quantitative details on temperature and concentration profiles of major and intermediate species and with various experimental studies; see for example $[4,5]$ to name a few.

Experimental studies of hydrocarbon combustion indicate that free electric charges, such as positive ions and/or electrons, are produced during the elementary reactions comprising the overall oxidation of practical fuels [6-9]. These observations point to the proposition that various global combustion characteristics, such as heat transfer rates, flame stabilization, flammability limits, and soot and smoke formation could be manipulated through electrical means. Understanding these effects requires some theoretical discussions, which have not been addressed in the literature and is the primary motivation of this work.

In one of the earliest works by Lewis [6] systematic measurements, using various fuels, were made to identify the effect of external fields on flame deflection and flame extinction. It was observed that for every type of fuel tested, the flame in an electric field appeared to have a charged behavior that would lead to its deflection towards the positive electrode and that the extent of the alterations in flame shape, burning rate and extinction limits depended not only upon the fuel type and equivalence ratio of the mixture but also on the strength of the field. Calcote $[7,10]$ reviewed the possible mechanisms leading to the creation of ions in flames, and concluded from his experimental results that chemi-ionization or cumulative excitation was the main reason for the production of these charged particles. Similarly, Goodings et al. [8,9] performed extensive experiments detailing the mechanism of charge creation and the different type of positive and negative ions produced in fuel-lean and rich methane flames, and suggested that chemi-ionization is the dom-

\footnotetext{
${ }^{1}$ The discussion in Cheatham and Matalon [3] is limited to equidiffusion flames, i.e., when the Lewis numbers of the fuel and oxidizer do not significantly differ from one. Unfortunately, this fact was not properly stated in the paper. Consequently, the statement on page 112 , that "to leading order the flame temperature is the adiabatic flame temperature for all Lewis numbers" is incorrect, and the curves corresponding to the extreme values of the Lewis number in Figure 6, may be beyond their range of validity.
} 
inant mechanism for generation of charged species. This conclusion was also verified more recently by a number of other studies; e.g., [11-13].

Recent investigations have reported on the effect of external electric fields on flame characteristics, for both premixed as well as diffusion flames. Transverse electric fields were used to modify the flame speed of various premixed air-fuel mixtures [14]. Droplet combustion studies showed the possibility of a change in flame shape and mass burning rate for sooting and non-sooting flames [15-18]. Droplet combustion experiments were also conducted to examine the coupling of electrostatics with combustion chemistry [19]. Experiments on lifted laminar flames showed that the application of $\mathrm{AC}$ electric fields could permit control of flame stabilization and reattachment and lead to enhancement/reduction in the propagation speed of the edge flames [20]. The stabilization characteristics under AC electric fields were also examined for laminar Bunsen flames showing an increase in the detachment velocity under applied AC field [21]. Numerical simulations of diffusion flame were carried out to examine the usage of electric fields to counteract buoyancy [22], and to control lift-off heights and flame stabilization of jet flames. More recently, Sanchez-Sanz et al. [23] suggested a four-step reduced chemical mechanism as a modified Zel'dovich two-step mechanism for premixed flames in order to study the effects of electric fields on flame speed. Reviews on the subject were given by Bradley [24] who provided an account of the modes of creation of ions and/or electrons, and by Heinsohn and Becker [25], and Fialkov [26] who also discussed possible impact of utilizing these ionic species to modify flame characteristics. They suggested that external fields could be used not only to extinguish flames, but also to stabilize them, modify flame speeds, use electrolytically charged fluids in fire-fighting etc.

An external electric field can have two major effects on a flame, namely an effect on chemical kinetics as well as the generation of a body force, and different views have been expressed regarding the mechanism responsible for the observed modifications of flame behavior by the applied field. Some argue that these modifications result from kinetic effects caused by the collision of charged and neutral particles, which is a consequence of the redistribution of the charged particles resulting from their ionic mobility and the direction of the applied electric field $[12,14]$. Others, $[15,16,18,19,22]$ argue that the body force due to the electric field is the primary mechanism responsible for the observed modifications of flame shape. The body forces can affect the combustion process in two ways. The first, often referred to as ionic wind, is through the momentum balance, and the second that we shall refer ${ }^{2}$ to as Ohmic heating is through the balance of energy resulting from the work, positive or negative, done by electrostatic forces. In practical terms, the ionic wind primarily modifies the pressure field depending upon the relative charge concentration in the system, whereas the Ohmic heating can act as a heat source or sink depending upon the ion distribution and the direction of the electric field. It has been argued that the ionic wind is the primary mechanism responsible for the modification of flame shape, with the effects of Ohmic heating negligible [22, 27].

The specific objective of this work is to develop a reduced chemical model that reflects the chemi-ionization process for general hydrocarbon fuels, and examine the effects of an externally applied electric field on diffusion flames. In particular, the characteristics of a spherical liquid drop steadily-burning in a quiescent oxidizer environment is considered. Since for this spherically symmetric configuration the effect of the ionic wind is simply to modify the pressure field, our study focuses exclusively on the effects of Ohmic heating and kinetic effects on the burning rate and the flame characteristics. In the following sections, we start by describing the general conservation equations for a chemically-reacting mixture that contains charged particles under the influence of an electric field. A reduced chemical mechanism is proposed in Sec. 3 to model the process of charge generation and destruction. With this basic framework in place, the governing equations for the steady spherically-symmetric burning of a liquid droplet are stated in Sec. 4 along with appropriate boundary conditions. The problem is addressed numerically and the results for no electric field and for strong and weak electric fields are summarized in Sec. 5-6, followed by conclusions in Sec. 7 .

\section{Governing Equations}

We consider a chemically reacting mixture consisting of overall $N$ species, that includes electrically charged species, in the presence of an external electric field $\mathbf{E}$. The mass balance of species $i$ is given by

$$
\rho \frac{D Y_{i}}{D t}=-\nabla \cdot\left(\rho Y_{i} \mathbf{V}_{i}\right)+\bar{\omega}_{i}
$$

where $\rho$ is the density of the mixture, $Y_{i}$ is the mass fraction, $\mathbf{V}_{i}$ the diffusion velocity and $\bar{\omega}_{i}$ the net rate of pro-

\footnotetext{
${ }^{2}$ Different names have been used in the literature, including Joule heating.
} 
duction of species $i$, with $i=1, \ldots, N$. The operator $D / D t=\partial / \partial t+\mathbf{v} \cdot \nabla$ is the convective derivative, where $\mathbf{v}$ is the bulk velocity and $t$ denotes time. Overall mass conservation implies

$$
\frac{\partial \rho}{\partial t}+\nabla \cdot(\rho \mathbf{v})=0
$$

Assuming the mixture behaves as a Newtonian fluid, the momentum conservation for the entire mixture is given by

$$
\rho \frac{D \mathbf{v}}{D t}=-\nabla p+\nabla \cdot \mu \boldsymbol{\Sigma}+\rho \mathbf{g}+\rho \sum_{i=1}^{N} Y_{i} \mathbf{f}_{\mathbf{i}}
$$

where $\Sigma$ is the rate of strain tensor and $\mu$ the shear viscosity of the mixture, $\mathrm{g}$ is the gravitational acceleration vector, and the last term on the right hand side represents the total body force resulting from the electrostatic forces acting on charged particles. The force per unit mass acting on a species $i$, is given by

$$
\mathbf{f}_{i}=s_{i}\left(q_{i} \mathcal{N}_{A} / W_{i}\right) \mathbf{E}
$$

where $\mathbf{E}$ is the electric field, $\mathcal{N}_{A}$ is Avogadro's constant, $q_{i}$ is the magnitude of the charge, $W_{i}$ is the molecular weight of species $i$, and

$$
s_{i}=\left\{\begin{array}{cl}
+1 & \text { if } i \text { is a positively charged species } \\
-1 & \text { if } i \text { is negatively charged species } \\
0 & \text { if } i \text { is a neutral species }
\end{array}\right.
$$

is a coefficient used to distinguish between the charged and the neutral species. When expressed in terms of the electric potential $\phi$, Gauss's law takes the form

$$
\nabla^{2} \phi=-\frac{1}{\varepsilon_{o}} \sum_{i=1}^{N} s_{i} q_{i} n_{i}
$$

where $\varepsilon_{O}$ the permittivity of free space and the introduction of $s_{i}$ implies that the sum accounts only for charged particles, and that the effective charge of species $i$ may be positive or negative. To classify the ion concentration, we note that the mass fraction of a charged species $Y_{i}$ is related to the number density through the relation $n_{i}=\left(\rho Y_{i} / W_{i}\right) \mathcal{N}_{A}$.

Neglecting radiation and diffusion (Dufour) effects in the heat flux vector, energy conservation for the entire mixture takes the form

$$
\begin{gathered}
\rho \frac{D h}{D t}=\nabla \cdot(\lambda \nabla T)-\nabla \cdot\left(\rho \sum_{i=1}^{N} h_{i} Y_{i} \mathbf{V}_{i}\right)+\frac{D p}{D t} \\
+\Phi+\rho \sum_{i=1}^{N} Y_{i} \mathbf{f}_{\mathbf{i}} \cdot \mathbf{V}_{i}
\end{gathered}
$$

where $h_{i}$ is the enthalpy of species $i$, with $h$ the enthalpy of the mixture, $T$ is the temperature and $\lambda$ the thermal conductivity of the mixture, $p$ is the pressure and $\Phi$ is the viscous dissipation function. The last term on the right hand side of this equation is total work done by electrostatic forces.

If the species are assumed to be ideal gases, the equation of state for the mixture may be expressed in the form

$$
p=\rho \mathcal{R} T / \bar{W}
$$

where $\bar{W}=\left(\sum_{i=1}^{N} Y_{i} / W_{i}\right)^{-1}$ is the mixture molecular weight with $W_{i}$ the molecular weight of species $i$, and $\mathcal{R}$ is the universal gas constant. The caloric equation of state is given by

$$
h=\sum_{i=1}^{N} Y_{i}\left(h_{i}^{o}+\int_{T^{o}}^{T} c_{p_{i}} d T\right)
$$

where $h_{i}^{o}$ is the enthalpy of formation of species $i$ at the reference temperature $T^{o}$, and $c_{p_{i}}$ the specific heat (at constant pressure) of species $i$.

\section{Diffusion Velocities}

Neglecting thermal diffusion (Soret diffusion) and pressure gradient effects, the Stefan-Maxwell relations that relates the diffusion velocities to species concentrations are given by

$$
\begin{aligned}
\nabla X_{i}=\sum_{j=1}^{N}\left(\frac{X_{i} X_{j}}{\mathcal{D}_{i j}}\right) & \left(\mathbf{V}_{j}-\mathbf{V}_{i}\right) \\
& +\left(\frac{\rho}{p}\right) \sum_{j=1}^{N} Y_{i} Y_{j}\left(\mathbf{f}_{i}-\mathbf{f}_{j}\right)
\end{aligned}
$$

where, $X_{i}$ is the mole fraction of species $i$ and $\mathcal{D}_{i j}$ the binary diffusion coefficients. This expression can be simplified if the reactants are assumed scarce in an abundant inert. Let $i=N$ represent the inert species, $X_{i} \ll 1$ and $Y_{i} \ll 1$ for $i=1, \ldots, N-1$ while $X_{N} \approx Y_{N} \approx 1$, and the dilute approximation of the Stefan-Maxwell relations 
1 yields

5 Since the molecular weight of the mixture $\bar{W} \approx W_{N}$, 6 the relation between mole and mass fractions $Y_{i}=$ $X_{i} W_{i} / W_{N}$ implies

$$
Y_{i} \mathbf{V}_{i}=-\mathcal{D}_{i} \nabla Y_{i}+\frac{q_{i} \mathcal{N}_{A} \mathcal{D}_{i} Y_{i}}{\mathcal{R} T} \mathbf{E}
$$

$$
\frac{\mathcal{D}_{i}}{\kappa_{i}}=\frac{k_{\mathrm{b}} T}{q_{i}}
$$

where $\kappa_{i}$ is the ionic mobility and $k_{\mathrm{b}}=\mathcal{R} / \mathcal{N}_{A}$ is Boltzmann constant. Equation (7) then takes the form

$$
\rho Y_{i} \mathbf{V}_{i}=\underbrace{-\rho \mathcal{D}_{i} \nabla Y_{i}}_{\rho Y_{i} \mathbf{V}_{i_{\text {diff }}}} \underbrace{-s_{i} \kappa_{i} \rho Y_{i} \nabla \phi}_{\rho Y_{i} \mathbf{V}_{i_{\text {drift }}}}
$$

The diffusion fluxes are thus given by Fick's law of binary diffusion (species $i$ - inert), modified for the charged species by what may be considered as an effective drift velocity $\mathbf{V}_{i_{\text {drift }}}= \pm \kappa_{i} \nabla \phi$. The sign, conventionally introduced to distinguish between mobilities of positively and negatively charged species, is effectively represented in (8) by $s_{i}$. The effect of the external electric field is therefore to modify the net diffusive flux for the charged species, increasing the diffusion velocity $\mathbf{V}_{i_{\text {diff }}}$ for positively charged and decreasing it for negatively charged species when $\nabla \phi>0$. This effect is reversed when $\nabla \phi<0$ with a decreasing flux for positively charged and an increasing flux for negatively charged species. A similar expression to (8) was proposed by Landau and Lifshitz [28] for diffusion of suspended particles in a fluid, and used in a similar context to ours in [12], among others.

\section{Ionic wind}

The body force in the momentum equation (3) arises from Coulomb force; the force generated because of induced magnetic fields is produced by very small current densities that result from chemi-ionization, and is therefore negligible [29], except perhaps during electri- is the sum of the individual forces acting on the ions

$$
\mathbf{F}_{\text {ionic }} \equiv \rho \sum_{i=1}^{N} Y_{i} \mathbf{f}_{i}=-\nabla \phi \sum_{i=1}^{N} s_{i} q_{i} n_{i},
$$

or the difference between the net force of positively and negatively charged species, is referred to as the ionic wind. It essentially modifies the pressure field near the reaction zone when an external electric field is applied. We note that $q_{i}$ is the total charge of species $i$, which is the charge of a single electron or a multiple thereof.

\section{Ohmic heating}

Using the diffusion law (8), the work done by the electric field as represented by the last term on the right hand side in (6), also known as Ohmic heating, may be expressed as cal breakdown. The net body force (per unit mass), which $\rho \sum_{i=1}^{N} Y_{i} \mathbf{f}_{i} \cdot \mathbf{V}_{i}=\rho \sum_{i=1}^{N} Y_{i} \mathbf{f}_{i} \cdot\left(\mathbf{V}_{i_{\text {drift }}}+\mathbf{V}_{i_{\text {diff }}}\right)=\Omega_{\text {drift }}+\Omega_{\text {diff }}$ and comprises of two terms. The first,

$$
\Omega_{\text {drift }}=(\nabla \phi)^{2} \sum_{i=1}^{N} s_{i}^{2} \kappa_{i} q_{i} n_{i},
$$

represents the work done by the electric field on each of the charged species by "pushing" them in the direction of electrostatic attraction. This term is always positive and acts as a heat source. The second term

$$
\Omega_{\text {diff }}=\rho \mathcal{N}_{A} \sum_{i=1}^{N} \frac{\mathcal{D}_{i}}{W_{i}} s_{i} q_{i}\left(\nabla \phi \cdot \nabla Y_{i}\right)
$$

represents the work done on molecules that move because of Fickian diffusion. This work can be either positive or negative, depending on the overall contributions of the charged species, which individually could make positive/negative work depending on whether the direction of the field is aligned with their direction of diffusion, i.e., depending on the sign of $\nabla \phi \cdot \nabla Y_{i}$. Thus, depending upon the flow configuration and the direction of the electric field, Ohmic heating acts as a heat source when it is positive, or as a heat sink, when it is negative.

\section{Simplified equations}

The gas velocities of deflagrative processes are typically much smaller than the speed of sound, implying that the burning process is under nearly-isobaric conditions. The variations in pressure from the ambient pressure $P_{\infty}$, 
that are needed to balance momentum, are small and on the order of the representative Mach number squared. Consequently, the density of the mixture is inversely proportional to temperature, and the pressure work and viscous dissipation in the energy equation are negligibly small. For simplicity we assume that all species have constant and equal specific heats; i.e., $c_{p_{i}}=c_{p}=$ const. for all $i$, the diffusion coefficients $\rho \mathcal{D}_{i}$ and the electric coefficients $\rho \kappa_{i}$ of all species are constant, and the viscosity $\mu$ and thermal conductivity $\lambda$ of the mixture are constants. We also assume that all charged particles carry the same charge in magnitude, $q_{i}=q$ and that the ionic mobilities $\kappa_{i}$ are equal for all $i$. For realistic flames, these properties are not constant and depend primarily on temperature. The governing equations then reduce to

$$
\begin{gathered}
\frac{\partial \rho}{\partial t}+\nabla \cdot(\rho \mathbf{v})=0 \\
\rho \frac{D \mathbf{v}}{D t}=-\nabla p+\mu \nabla \cdot \boldsymbol{\Sigma}+\rho \mathbf{g}+\mathbf{F}_{\text {ionic }} \\
\rho \frac{D Y_{i}}{D t}=\bar{\omega}_{i}+\rho \mathcal{D}_{i} \nabla^{2} Y_{i}+s_{i} \rho \kappa_{i} \nabla \cdot\left(Y_{i} \nabla \phi\right) \\
\rho c_{p} \frac{D T}{D t}=\lambda \nabla^{2} T-\sum_{i=1}^{N} h_{i}^{o} \bar{\omega}_{i}+\Omega_{\mathrm{drift}}+\Omega_{\mathrm{diff}} \\
\nabla^{2} \phi=-\frac{q}{\varepsilon_{o}} \sum_{i=1}^{N} s_{i} n_{i} \\
P_{\infty}=\rho \mathcal{R} T / \bar{W}
\end{gathered}
$$

\section{Chemical Mechanism}

The complex reactions that comprise the oxidation of hydrocarbon fuels lead to the creation of ions and electrons in non-negligible concentrations. Amongst the different sources of creation of charged species from the reaction of the neutral molecules, chemi-ionization is considered the dominant mode in hydrocarbon combustion [7]. It was determined that $\mathrm{CH}$ radicals are the main precursor to these reactions, and the following three elementary reactions

$$
\begin{array}{ll}
\text { (A) } & \mathrm{CH}+\mathrm{O} \longrightarrow \mathrm{CHO}^{+}+\mathrm{e}^{-} \\
\text {(B) } & \mathrm{CHO}^{+}+\mathrm{H}_{2} \mathrm{O} \longrightarrow \mathrm{CO}+\mathrm{H}_{3} \mathrm{O}^{+} \\
\text {(C) } & \mathrm{H}_{3} \mathrm{O}^{+}+\mathrm{e}^{-} \longrightarrow \mathrm{H}_{2} \mathrm{O}+\mathrm{H}
\end{array}
$$

are among the most important one for the creation of ions $[7,30]$. The first reaction describes generation of charge from neutral reactants through electron transfer, forming a positive ion and an electron, or negative ion. The second step is an ionic rearrangement reaction. The last step is a recombination reaction, whereby charged particles react to give neutral products. Although the first step leading to $\mathrm{CHO}^{+}$is believed to be responsible for primary ionization, it has been experimentally found that hydronium ion, $\mathrm{H}_{3} \mathrm{O}^{+}$, is the dominant positive ion in flames. And although electrons are the main carrier of negative charge $[8,29]$, species like $\mathrm{O}_{2}^{-}$have been also observed, but their number concentrations are typically small [9].

Keeping these into consideration, we propose the following generic three-step mechanism to mimic the generation of chemi-ions during the oxidation of hydrocarbon fuels.

$$
\begin{aligned}
\nu_{F} \mathrm{~F}+\nu_{O} \mathrm{O} & \longrightarrow \mathrm{X}_{1}+\mathrm{X}_{2}+\left\{\hat{Q}_{1}\right\} \\
X_{2} & \longrightarrow \mathrm{I}_{\mathrm{p}}+\mathrm{I}_{\mathrm{n}} \\
\mathrm{I}_{\mathrm{p}}+\mathrm{I}_{\mathrm{n}}+\mathrm{X}_{1} & \longrightarrow\left(\nu_{F}+\nu_{O}\right) \mathrm{P}+\left\{\hat{Q}_{3}\right\}
\end{aligned}
$$

Here, $\mathrm{F}$ and $\mathrm{O}$ stand for the chemical species acting as fuel and oxidizer, respectively, and $\mathrm{P}$ represents the final products. Step (1) is an initiation reaction that produces two radical groups, $\mathrm{X}_{1}$ and $\mathrm{X}_{2}$ and releases an amount $\hat{Q}_{1}$ of heat. Step (2) involves chain breaking of radicals from the $\mathrm{X}_{2}$ group, and the creation of positive $\mathrm{I}_{\mathrm{p}}$ and negative $I_{n}$ ionic species. Step (3) is a recombination reaction that releases an amount $\hat{Q}_{3}$ of heat. The radical group $\mathrm{X}_{1}$ is responsible for the oxidation of fuel to $\mathrm{CO}$ and its subsequent conversion to $\mathrm{CO}_{2}$, while the second group, $\mathrm{X}_{2}$, contains the radicals responsible for the formation of positive and negative ions. For the formation of ions, the crucial element is the $\mathrm{CH}$ radical and since carbon atoms originate from the fuel, it is reasonable to presume that the involvement of the fuel is essential for the creation of the radical $X_{2}$. Hence the group of radicals $X_{2}$ originates from the fuel and is therefore dependent on the fuel type. On the other hand the group $X_{1}$ can be formed from a combination of both, fuel and oxidizer.

This proposed mechanism was inspired by the work of Peters [31] who used a systematic series of steady-state and partial-equilibrium assumptions to suggest a four-step mechanism for methane oxidation. This mechanism was broken down into an oxidation step leading to $\mathrm{CO}$, two chain branching steps and a fourth reaction representing the conversion of $\mathrm{CO}$ to $\mathrm{CO}_{2}$, thereby releasing majority of the heat of the entire process. We note that similar reduced-step mechanisms can be written for the oxidation of most hydrocarbons but, due to their complex structure, the accuracy of the models would depend on the validity of the assumptions of steady-state and partial-equilibrium used in their derivation. 
As noted in the proposed scheme, heat is released in the first and third steps only in amounts $\hat{Q}_{1}$ and $\hat{Q}_{3}$ (per unit mass). The justification for this comes from the fact that step (2) behaves like a chain propagation step responsible only for the creation of ions. The rates of steps (1) and (3) are temperature sensitive with activation energies $E_{1}>E_{3}$, whereas step (2) is assumed to have practically a zero activation energy. A similar mechanism to ours, but involving four steps, was suggested by Sanchez-Sanz et al. [23] and used to examine the effect of an electric field on premixed flames. In their model the heat release was also assumed to occur during the product formation.

\section{Droplet Configuration}

We consider the spherically-symmetric burning of a well-mixed liquid fuel droplet of radius $r_{0}$ and uniform temperature, in the absence of gravity (i.e. $\mathbf{g}=0$ ), and subjected to an external electrical field, as shown schematically in Fig 1. The idealization may correspond to a spherical porous sphere internally supplied with liquid fuel, to a liquid droplet suspended on a quartz fiber, or to a freely falling fuel droplet all under microgravity conditions. It is assumed that the droplet surface is kept at a constant, externally imposed voltage of magnitude $\mathbb{V}$, whereas the voltage far away from the droplet is zero. Due to the disparity between the liquid and gas densities, the burning process may be considered quasi-steady. Our objective is to examine the effect of the electric field on the steady burning and, in particular, its effect on the flame standoff distance and the mass burning rate.

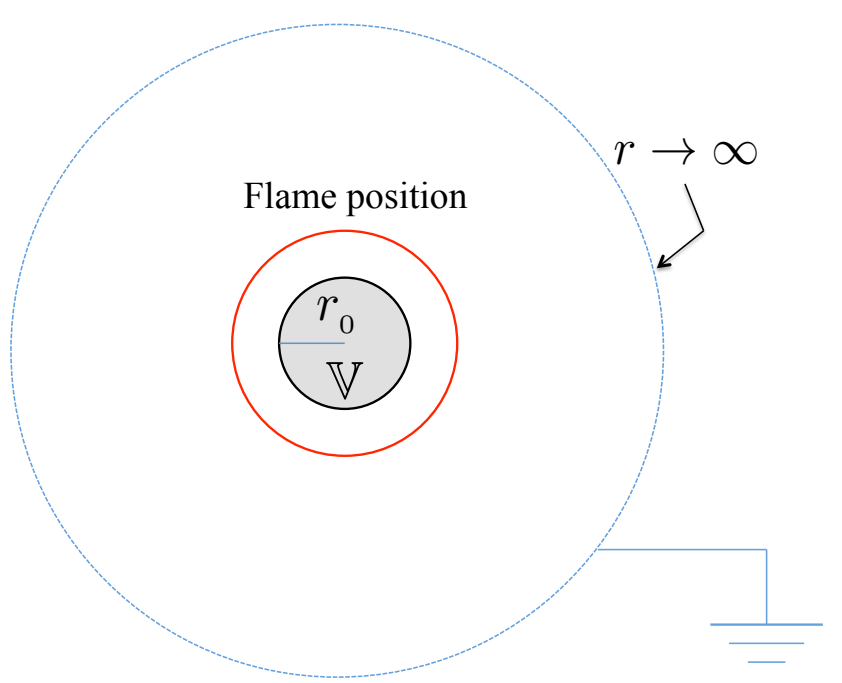

Figure 1: Schematic of the fuel droplet problem in the presence of an electric field.
We introduce dimensionless variables

$$
\begin{aligned}
& r^{*}=\frac{r}{r_{o}} \quad \rho^{*}=\frac{\rho}{\rho_{\infty}} \quad v^{*}=\frac{v}{\mathcal{D}_{t h} / r_{o}} \\
& \phi^{*}=\frac{\phi}{\mathbb{V}} \quad T^{*}=\frac{T}{T_{\infty}} \quad P^{*}=\frac{P}{P_{\infty}},
\end{aligned}
$$

using as reference the ambient conditions $\rho_{\infty}, P_{\infty}$ and $T_{\infty}$, the droplet radius $r_{0}$, the diffusion velocity $\mathcal{D}_{t h} / r_{0}$, and the voltage $\mathbb{V}$; here $\mathcal{D}_{t h}=\lambda / \rho c_{p}$ the thermal diffusivity of the mixture. With the adopted simplifications and non-dimensionalization, the governing equations reduce (after dropping the ${ }^{*}$ from the dimensionless variables for simplicity of notation) as follows.

The overall conservation of mass

$$
\frac{d}{d r}\left(r^{2} \rho v\right)=0
$$

implies that $r^{2} \rho v=M$, where $M$ denotes the constant (dimensionless) mass flow rate, or burning rate. The mass balance equations (11) for the various species, become for the proposed scheme

$$
\begin{gathered}
\frac{M-2 r}{r^{2}} \frac{d Y_{F}}{d r}-L e_{F}^{-1} \frac{d^{2} Y_{F}}{d r^{2}}=-\omega_{1} \\
\frac{M-2 r}{r^{2}} \frac{d Y_{O}}{d r}-L e_{O}^{-1} \frac{d^{2} Y_{O}}{d r^{2}}=-\nu \omega_{1} \\
\frac{M-2 r}{r^{2}} \frac{d Y_{X_{1}}}{d r}-L e_{X_{1}}^{-1} \frac{d^{2} Y_{X_{1}}}{d r^{2}}=\nu_{1}\left(\omega_{1}-\omega_{3}\right) \\
\frac{M-2 r}{r^{2}} \frac{d Y_{X_{2}}}{d r}-L e_{X_{2}}^{-1} \frac{d^{2} Y_{X_{2}}}{d r^{2}}=\nu_{2}\left(\omega_{1}-\omega_{2}\right) \\
\frac{M-2 r}{r^{2}} \frac{d Y_{p}}{d r}-L e_{p}^{-1} \frac{d^{2} Y_{p}}{d r^{2}}=\nu_{p}\left(\omega_{2}-\omega_{3}\right) \\
\frac{\eta_{p} \frac{1}{r^{2}} \frac{d}{d r}\left(r^{2} Y_{p} \frac{d \phi}{d r}\right)}{r^{2}} \frac{d Y_{n}}{d r}-L e_{n}^{-1} \frac{d^{2} Y_{n}}{d r^{2}}=\nu_{n}\left(\omega_{2}-\omega_{3}\right) \\
-\eta_{n} \frac{1}{r^{2}} \frac{d}{d r}\left(r^{2} Y_{n} \frac{d \phi}{d r}\right)
\end{gathered}
$$

where $Y_{F}$ and $Y_{O}$ are the mass fractions of the fuel and oxidizer, $Y_{X_{1}}$ and $Y_{X_{2}}$ the mass fractions of the radicals, and $Y_{p}$ and $Y_{n}$ the mass fractions of the positive and negative ions, respectively, with the reaction rates given by

$$
\begin{gathered}
\omega_{1}=D a_{1} \rho^{2} Y_{F} Y_{O} e^{-\beta_{1} / T}, \quad \omega_{2}=D a_{2} \rho Y_{X_{2}}, \\
\omega_{3}=D a_{3} \rho^{3} Y_{p} Y_{n} Y_{X_{1}} e^{-\beta_{3} / T} ;
\end{gathered}
$$


the index here denotes the elementary step in the reaction scheme. The mass-weighted stoichiometric coefficients (defined relative to the fuel) appearing the these equations are

$$
\begin{gathered}
\nu=\frac{\nu_{O} W_{O}}{\nu_{F} W_{F}}, \quad \nu_{1}=\frac{W_{X_{1}}}{\nu_{F} W_{F}}, \quad \nu_{2}=\frac{W_{X_{2}}}{\nu_{F} W_{F}}, \\
\nu_{p}=\frac{W_{p}}{\nu_{F} W_{F}}, \quad \nu_{n}=\frac{W_{n}}{\nu_{F} W_{F}} .
\end{gathered}
$$

The energy equation (12) takes the form

$$
\frac{M-2 r}{r^{2}} \frac{d T}{d r}-\frac{d^{2} T}{d r^{2}}=Q_{1} \omega_{1}+Q_{3} \omega_{3}+\chi\left(\Omega_{\mathrm{drift}}+\Omega_{\mathrm{diff}}\right)
$$

where

$$
\begin{aligned}
\Omega_{\mathrm{drift}} & =\rho\left(\frac{d \phi}{d r}\right)^{2}\left(\eta_{p} Y_{p}+\frac{\nu_{p}}{\nu_{n}} \eta_{n} Y_{n}\right), \\
\Omega_{\mathrm{diff}} & =\rho\left(\frac{d \phi}{d r}\right)\left(\frac{d Y_{p}}{d r}-\frac{\nu_{p}}{\nu_{n}} \frac{d Y_{n}}{d r}\right),
\end{aligned}
$$

and the equation of state (14) determines the density $\rho=$ $1 / T$. Gauss's equation (13) becomes

$$
\frac{1}{r^{2}} \frac{d}{d r}\left(r^{2} \frac{d \phi}{d r}\right)=-\alpha \rho\left(Y_{p}-\frac{\nu_{p}}{\nu_{n}} Y_{n}\right) .
$$

The momentum equation (10), which is uncoupled from the rest of the equations and is used a-posteriori to determine the pressure field, will be discussed later.

The dimensionless parameters in these equations include:

$$
L e_{i}=\mathcal{D}_{t h} / \mathcal{D}_{i}, \quad \eta_{i}=\rho \kappa_{i} \mathbb{V} / \rho \mathcal{D}_{i}
$$

corresponding respectively to the Lewis numbers representing the ratio of the thermal-to-mass diffusivities of species $i$, and the ratio of electrical-to-mass diffusivities of the charged particles $i=p, n$; the heat release parameters

$$
Q_{1}=\frac{\hat{Q}_{1} / c_{p} T_{\infty}}{\nu_{F} W_{F}}, \quad Q_{3}=\frac{\hat{Q}_{3} / c_{p} T_{\infty}}{\nu_{F} W_{F}}
$$

the parameters associated with the electric field

$$
\alpha=q \rho_{\infty} r_{o}^{2} \mathcal{N}_{A} / \varepsilon_{o} W_{p} \mathbb{V}, \quad \chi=q \mathcal{N}_{A} \mathbb{V} / c_{p} T_{\infty} W_{p}
$$

corresponding respectively to the ratio of the permittivity of the medium to that of vacuum, which is a measure of the strength of the electric field, and the ratio of the electrical to the ambient energy, or the Ohmic heating parameter; the activation energy parameters $\beta_{1}=E_{1} / R T_{\infty}$ and $\beta_{3}=E_{3} / R T_{\infty}$ and the Damköhler numbers

$$
\begin{gathered}
D a_{1}=\frac{\nu_{F} \rho_{\infty}^{2} r_{o}^{2} B_{1}}{\left(\lambda / c_{p}\right) W_{O}}, \quad D a_{2}=\frac{\nu_{F} W_{F} \rho_{\infty} r_{o}^{2} B_{2}}{\left(\lambda / c_{p}\right) W_{2}}, \\
D a_{3}=\frac{\nu_{F} W_{F} \rho_{\infty}^{3} r_{o}^{2} B_{3}}{\left(\lambda / c_{p}\right) W_{p} W_{n} W_{X_{1}}}
\end{gathered}
$$

representing the ratios of the diffusion to the reaction time of the three elementary steps, with $B_{i}$ the corresponding pre-exponential factors.

The system of equations (16)-(22) must be solved subject to the following boundary conditions.

At the droplet surface $r=1$ :

The net mass flowing out of the droplet surface consists only fuel, implying

$$
\begin{gathered}
M Y_{F}-\frac{d Y_{F}}{d r}=M \\
M Y_{i}-\frac{d Y_{i}}{d r}=0 \quad \text { for } Y_{i}=Y_{O}, Y_{X_{1}}, Y_{X_{2}} .
\end{gathered}
$$

Assuming that the current caused by the external field is very small, we stipulate that ions in the gas phase do not penetrate the droplet, such that

$$
M Y_{p}-\frac{d Y_{p}}{d r}=0, \quad M Y_{n}-\frac{d Y_{n}}{d r}=0
$$

The prescribed voltage at the droplet surface yields

$$
\phi=1
$$

If the droplet temperature is maintained constant and uniform, an energy balance at the droplet surface implies that all the heat conducted back to the droplet goes into evaporating the liquid fuel, namely.

$$
\frac{d T}{d r}=M L_{\mathrm{v}}
$$

where $L_{\mathrm{v}}$ is the (dimensionless) latent heat of vaporization. The dependence of the fuel vapor partial pressure on temperature at the droplet surface is typically expressed by the Claussius Clapeyron relation, but for simplicity we shall assume here that the surface temperature remains fixed, namely,

$$
T=T_{s}
$$

Far away from the droplet, as $r \rightarrow \infty$ :

The state of the gas and voltage are specified and the am- 
bient contains only oxidizer. Then

$$
\begin{gathered}
\rho=T=1, \quad Y_{O}=Y_{O \infty}, \quad \phi=0 \\
Y_{F}=Y_{p}=Y_{n}=Y_{X_{1}}=Y_{X_{2}}=0
\end{gathered}
$$

The eight second order differential equations require only sixteen boundary conditions; the additional condition is needed to determine the burning rate $M$.

In the following, this system of equations will be solved for the case of unity Lewis numbers $L e_{i}=1$ for all $i$, equal mobility, i.e., $\eta_{i}=\eta$ for $i=p, n$, with stoichiometric coefficients equal to one, except for the fuel-tooxidizer ratio $\nu$; i.e. $\nu_{i}=1$ for $i=1,2$ and $i=p, n$. Under these conditions, examination of the governing equations reveals that the system of equations is symmetric with respect to the applied potential, i.e., the application of a negative potential at the droplet surface would result in the same flame behavior as with that of a positive potential because, upon application of a negative potential, the positive and negative ions will merely switch their identities. Practically however, in situations where there is considerable difference between the properties of the ionic species, for example when the electrons form the majority of the negative charge carriers, distinct properties (Lewis number, molecular weight and electrical mobility) between species may cause the flame to behave differently for positive/negative applied potential.

The remaining parameters include the charged particles mobility $\eta$, the heat release and latent heat $Q_{1}, Q_{3}$ and $L_{\mathrm{v}}$, the Ohmic heating parameter $\chi$, the droplet surface temperature $T_{s}$ and the oxidizer mass fraction at the ambient state $Y_{O_{\infty}}$. In the results presented below we have fixed

$$
\begin{gathered}
Q_{1}=Q_{3}=50, \quad \beta_{1}=50, \quad \beta_{3}=10 \\
\nu=3.52, \quad Y_{O_{\infty}}=0.233, \quad L_{\mathrm{v}}=0.658, T_{s}=1.23
\end{gathered}
$$

choosing n-heptane as the representative fuel, noting that variation of these parameters will not significantly affect the qualitative nature of our analysis. The choice of $Q_{1}=$ $Q_{3}$ was selected as representative, as discussed below. A judicious estimate of the parameters yields the following magnitude of the Damköhler numbers: $D a_{1} \sim 10^{8}$, $D a_{2} \sim 2.5 \cdot 10^{2}$, and $D a_{3} \sim 0.4$. It is convenient, however, to rescale the Damköhler numbers in the computations by introducing

$$
\begin{gathered}
D_{1}=D a_{1} \beta_{1}^{-3} e^{-\beta_{1} / T_{a}}, \quad D_{2}=D a_{2} \beta_{1}^{-2}, \\
D_{3}=D a_{3} \beta_{3}^{-1} e^{-\beta_{3} / T_{a}}
\end{gathered}
$$

where $T_{a}$ is the adiabatic flame temperature correspond- ing to complete combustion, in the absence of an electric field. This scaling is suggested from the asymptotic treatment of a single step reaction mechanism for large activation energies [3]. The presumption here is that the initiation and chain breaking steps (1) and (2) occur on a much shorter time scale, such that they are both confined to a relatively thin region where the temperature is near $T_{a}$, whereas the recombination step (3) occurs across the entire field. For a given fuel, the Damköhler numbers are controlled by the system pressure and/or the droplet size and it is of interest to examine the variations of the flame behavior over a wide range of their values. We choose to let $D_{1}$ vary, and specify the ratios $D_{2} / D_{1}$ and $D_{3} / D_{1}$ to reflect the relative magnitudes of $D a_{2} \gg D a_{3}$. Here we have taken $D_{2} / D_{1}=0.25$, and $D_{3} / D_{1}=0.0125$, as representative.

With the exception of $\alpha$, the two parameters directly affected by the imposed voltage $\mathbb{V}$ at the surface of the droplet are $\chi$ and $\eta$. Although they appear as independent parameters, their ratio, $\chi / \eta=\mathcal{R} / c_{p} W_{p}$ depends only on the mixture properties and may be treated as a constant $c$, say. Most of the results presented below assume $c=10$, retaining $\chi$ as the free parameter.

A pseudo time relaxation finite difference scheme is used to integrate the governing equations numerically. A fourth-order explicit central difference formula is used for spatial derivatives with fourth-order forward/backward approximations at the boundaries $r=1$ and $r=R$, respectively, with $R$ selected sufficiently large to mimic the far field appropriately. The integration in time was carried out with an explicit fourth-order Runge-Kutta method, and extended until the solution relaxes to a steady state. The convergence criterion was based on a tolerance of $0.05 \%$, examined after every physical time unit.

\section{No External Electric Field}

In the following sections, the case of no external electric field is first discussed and the prediction of the proposed three-steps reaction scheme is examined in comparison to the classical results based on a single-step chemistry model. With $\chi=\eta=0$, the governing equations reduce to a reaction-diffusion system for the proposed threestep reaction mechanism. When the Damköhler number $D a_{1} \gg 1$, a direct implication from eqs. (16) and (17) is that the initiation reaction (1) is confined to a thin reaction zone that separates the region near the droplet where there is fuel but no oxidizer, i.e., where $Y_{O} \equiv 0$, from the region behind the reaction sheet where there is only oxidizer and $Y_{F} \equiv 0$, much like the Burke-Schumann limit of a classi- 
cal one-step global reaction. Since the radical $X_{2}$ is also produced very fast (at the same rate that the fuel and oxidizer are consumed) and is consumed very fast (at a rate that depends only on its own concentration), it is reasonable to expect that it is in "steady-state", namely $\omega_{1} \approx \omega_{2}$, such that $Y_{X_{2}}=0$ everywhere, except in the reaction zone where it exists in small quantities. In contrast, the radical $X_{1}$ and the charged species although produced in the reaction zone, are transported away upon their creation and are therefore consumed at a much slower rate throughout the entire field. This flame structure is reflected in the profiles shown in Fig. 2 calculated for $D_{1}=0.8$, corresponding to a sufficiently large $D a_{1}$, with all other parameters, as specified above. We see that the flame (or reaction sheet) stands at $r \approx 28$ (units of length), the fuel and oxidizer diffusing from opposing ends are completely consumed at the flame, and the temperature reaches its maximum value $T_{a}=4.4$ there. It must be mentioned here that similar to the classical droplet theory, the flame radius predicted above is larger than observed experimental values and is believed to result from the assumption of unity Lewis number [32]. While the radical $X_{2}$ is present only inside this reaction layer in small amount, the radical $X_{1}$ and the charged species exist in the entire domain. We note that for unity Lewis numbers and mass-weighted stoichiometric coefficients, the mass fractions $Y_{X_{1}}=Y_{p}=Y_{n}$.

We note that equations (16)-(17) for the fuel and oxidizer mass fractions, and eq. (22) for the temperature, are the exact equations that need to be solved for a one-step chemistry model, provided all the heat is generated in the initiation reaction (1), i.e., when $Q_{3}=0$. The remaining equations (18)-(21) merely determine the generation of the intermediate species and their distribution across the combustion field. When the total heat $Q=Q_{1}+Q_{3}$ is partially released in the initiation step $\left(Q_{1}\right)$ and partially in the recombination step $\left(Q_{3}\right)$, the production and consumption of the radicals $X_{1}$ play an important role, being intimately related to the rate of consumption of fuel and oxidizer. One observes that the coupling function $T+Q Y_{O} / \nu+Q_{3} Y_{X_{1}}$ is a conserved scalar ${ }^{3}$ that satisfies

$$
\begin{aligned}
& T+Q Y_{O} / \nu+Q_{3} Y_{X_{1}}= \\
& \quad 1+Q Y_{O \infty} / \nu+e^{M}\left(L_{\mathrm{v}}+Q_{3} Y_{X_{1 s}}\right)\left(e^{-M / r}-1\right)
\end{aligned}
$$

where $Y_{X_{1 s}}$ is the value of the mass fraction of the radicals $X_{1}$ at the droplet surface $r=1$. In deriving this expression, the mass fraction of oxidizer at the droplet surface

${ }^{3}$ This is no longer true in the presence of an electric field because of Ohmic heating contribution in the energy equation.

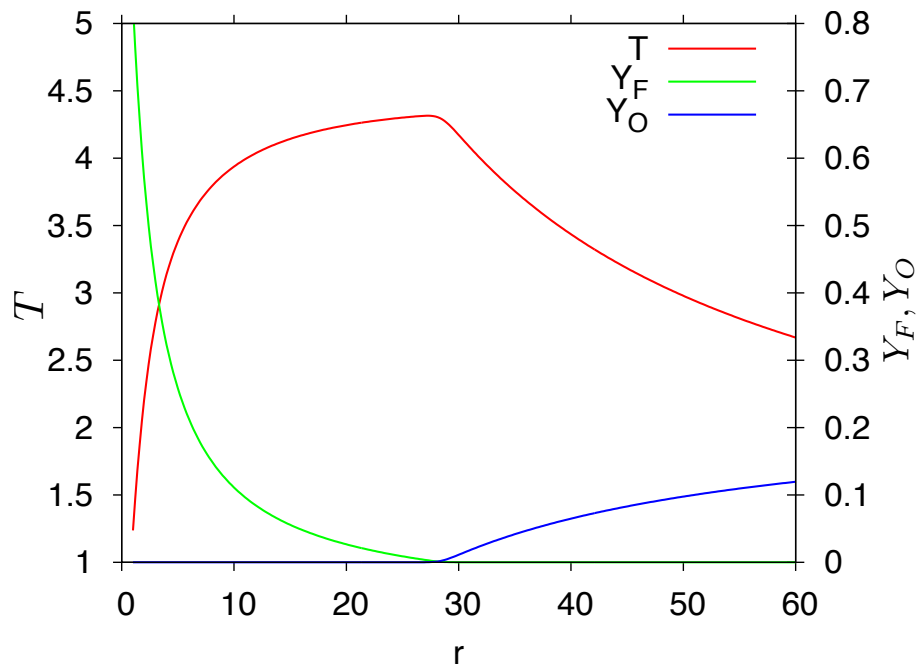

(a) Temperature, and fuel/oxidizer mass fractions

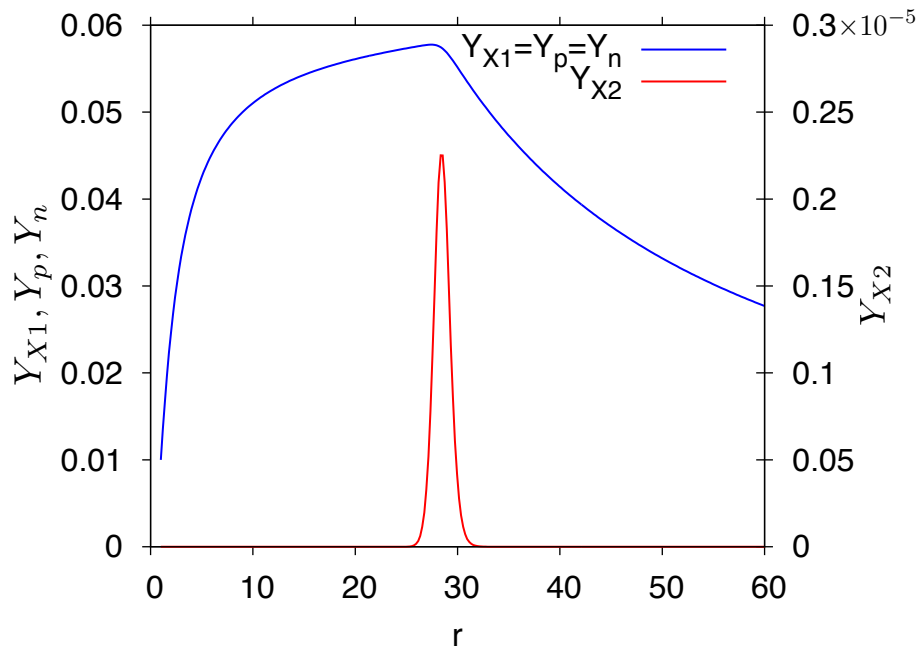

(b) Mass fraction of radicals and charged particles

Figure 2: Temperature and species mass fraction profiles across the combustion field, in the absence of an external electric field.

was taken equal to zero, which is a valid assumption when $D a_{1} \gg 1$, since all the oxidizer is consumed in a thin reaction remote from the droplet. The mass fraction of the radical $X_{1}$, however, is unknown and must be determined as part of the entire solution. Nevertheless, when (25) is evaluated at $r=1$, an expression for the mass burning rate is obtained in the form

$$
M=\ln (1+B),
$$

where

$$
B=\frac{1-T_{s}+Q Y_{O \infty} / \nu-Q_{3} Y_{X_{1 s}}}{L_{\mathrm{v}}+Q_{3} Y_{X_{1 s}}}
$$

is the "transfer number", or the ratio of the impetus-to- 


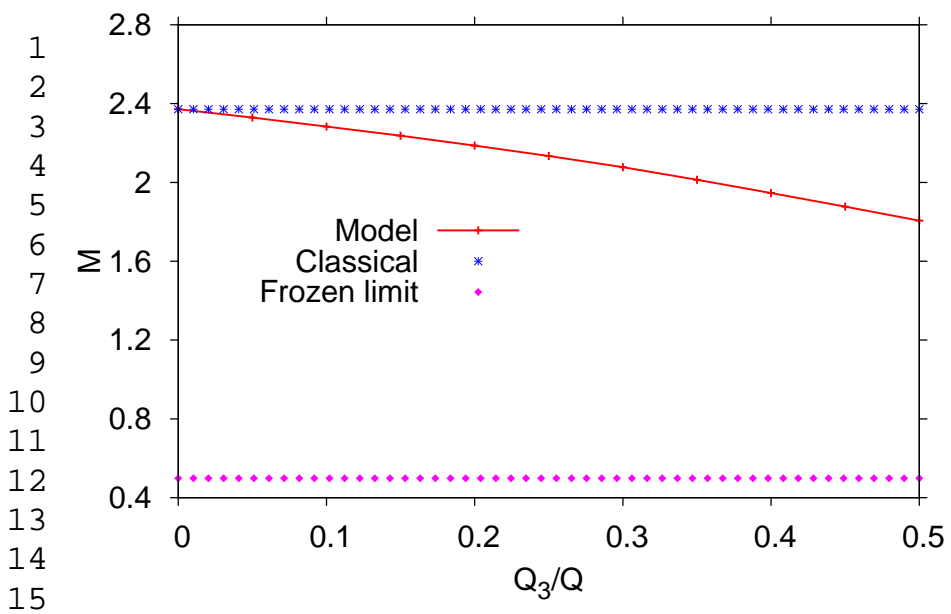

16

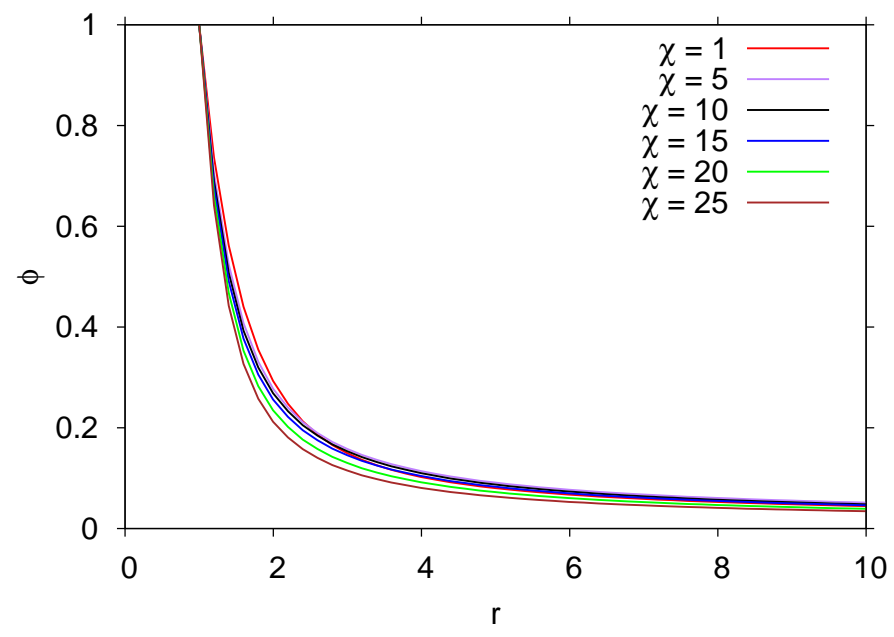

Figure 4: Variation in the electric potential $\phi$ across the combustion field for various values of the Ohmic heating parameter $\chi$, in the weak field limit.

large, namely when the internal barrier field created by the charged particles generated by the chemical reactions is too weak, or sufficiently strong to modify the imposed external field. Since the two important effects associated with the electric field, the ionic wind and Ohmic heating depend only on the effective charge at each point in the field, their effect on the behavior of the system in general circumstances could be deduced from our understanding of these two extreme limits.

In the strong field limit, i.e., $\alpha \ll 1$; the externally applied field is presumed strong enough and determined by Laplace's equation, with the potential field given, to leading order, by

$$
\phi \sim 1 / r
$$

for all values of $\chi$. The electric field remains effectively unaffected by the internal charge distribution due to chemi-ionization. In contrast, in the weak field limit, i.e., $\alpha \gg 1$, the internal charge distribution of the ionic species is sufficiently strong to create an internal repulsive barrier that can significantly affect the electric field distribution. In this limit Poisson's equation (23) is singular, implying that the imposed potential drops rapidly in a boundary layer near the droplet surface, outside of which it is effectively determined by the imposed external field. This behavior is reflected in Fig. 4, obtained by setting $\alpha=10^{3}$, for various values of $\chi$. The electric potential is modified primarily in the immediate vicinity of the droplet surface as the applied voltage increases.

A direct implication from equation (23) is that the concentrations of positive and negative charged species, in the weak field limit $(\alpha \gg 1)$, are proportional to each other; in our case since $\nu_{p}$ and $\nu_{n}$ were assumed equal, 


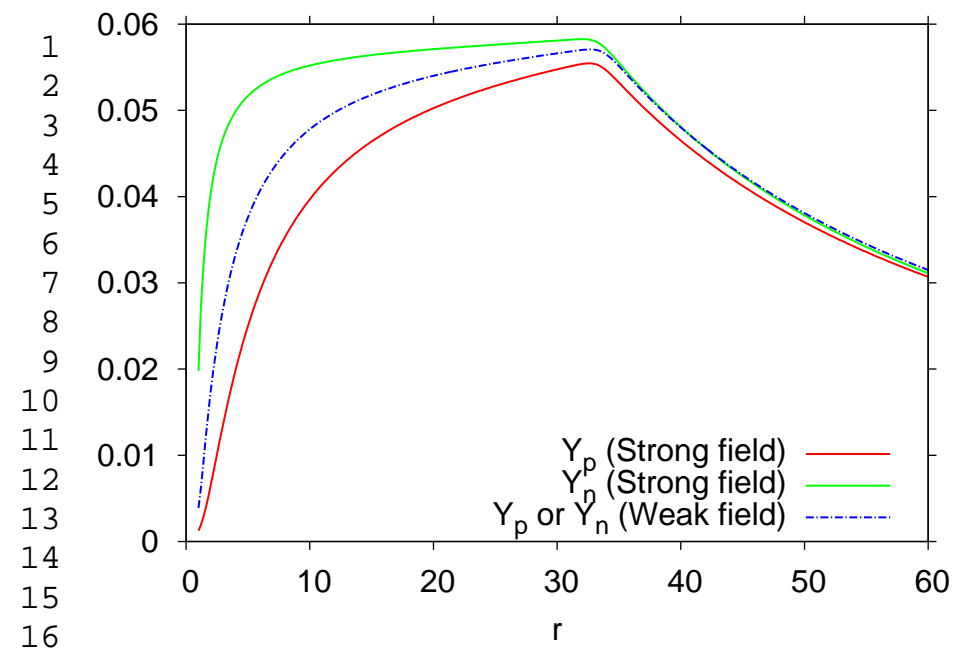

17

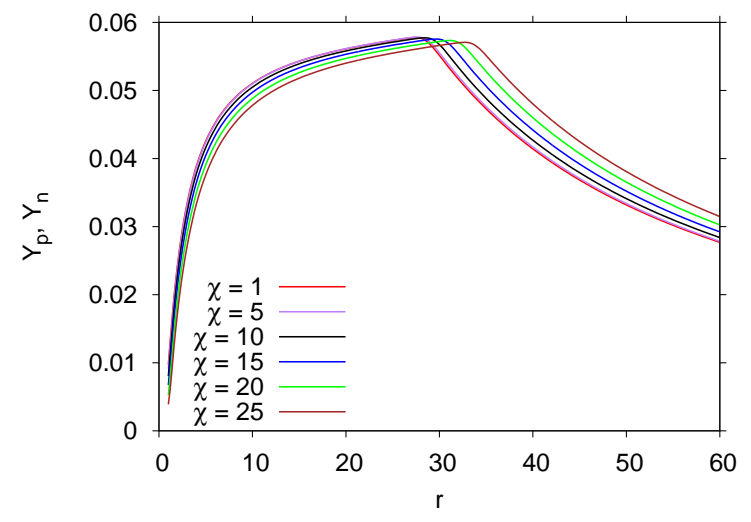

(a) $Y_{p}=Y_{n}$ in the weak field limit

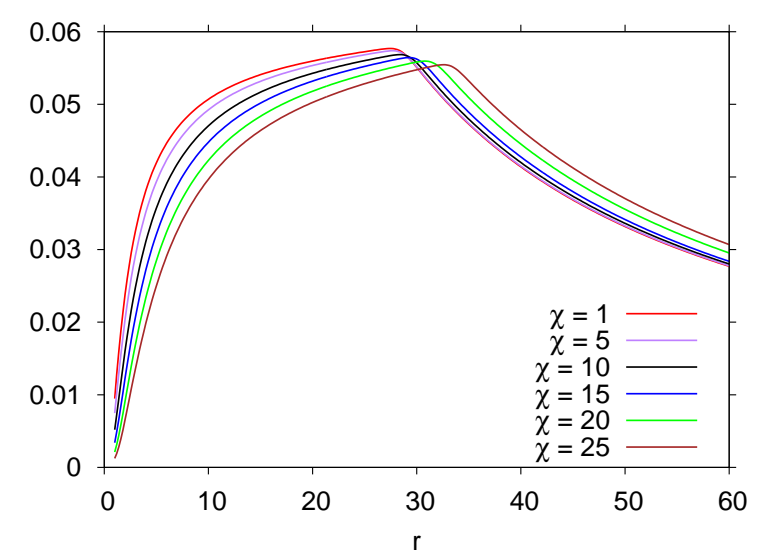

(b) $Y_{p}$ in strong field limit

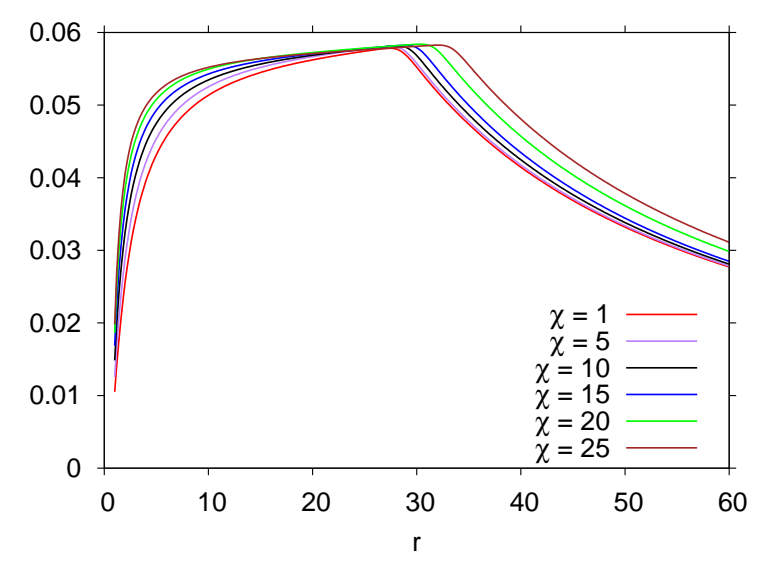

(c) $Y_{n}$ in strong field limit

Figure 6: Profiles of the mass fraction of positively and negatively charged species in the weak and strong field limits, for various values of the Ohmic heating parameter $\chi$.

is in a direction that opposes the electric field) and behaves as a heat sink. The balance of these two contributions is positive such that the Ohmic heating acts in effect as an energy source that causes an increase in the flame temperature, as shown in Fig. 7(a). The additional heat conducted to the droplet enhances the vaporization rate 


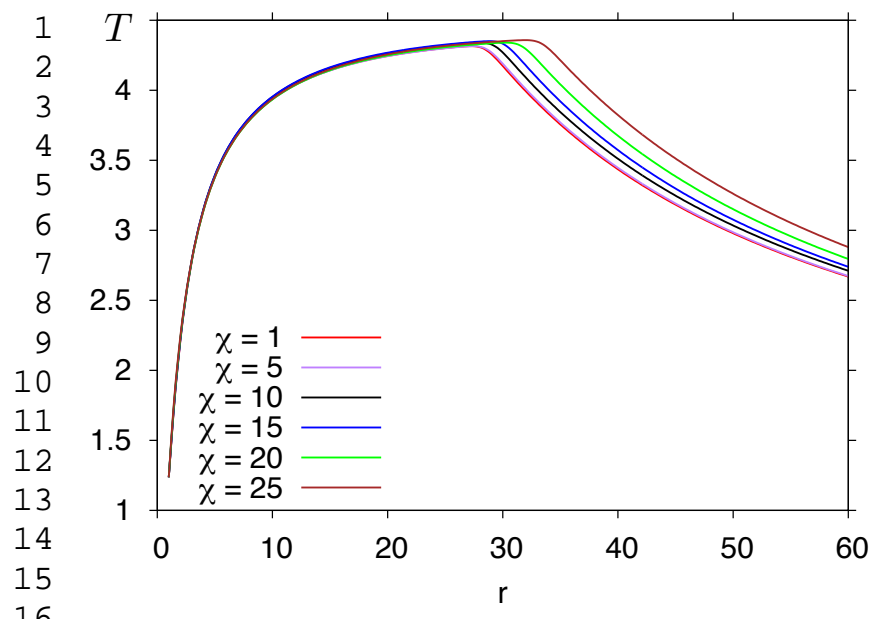

(a) $T$ in the combustion field

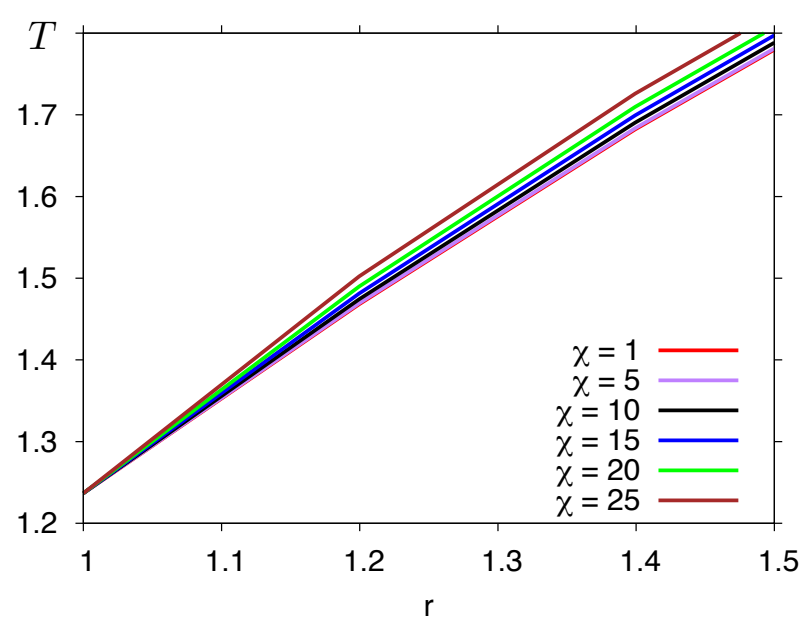

(b) $T$ near the droplet surface

Figure 7: Profiles of the temperature for various values of the Ohmic heating parameter $\chi$.

shown in Fig. 7(b) and increases the flux of fuel outwards which, as a result, causes the flame to move downstream towards the oxidizer. The translation of the flame downstream can be easily seen in Fig. 8. The mass fraction of the fuel and oxidizer are plotted for different values of $\chi$ and the reaction zone corresponds to the region where the fuel and oxidizer co-exist. Since the Ohmic heating is proportional to $\nabla \phi$ that does not vary much with $\chi$ at the location where the initiation reaction occurs, the temperature, fuel and oxidizer mass fractions and flame standoff distance are practically independent of the two limits of the electric field, strong and weak. Small variations may be encountered when the flame moves closer to the droplet surface because of the small increase in the induced electric field with $\chi$, but these are not of great significance.

The mass fraction of the radicals $X_{1}$ and $X_{2}$, and of the fuel and oxidizer, are not directly affected by the electric field, except for a translation resulting from the displacement of the flame with increasing $\chi$. This is shown in Fig. 9 to be valid in both limits, of weak and strong fields.

The overall positive contribution of the Ohmic heating effect leads to an increase in the mass burning rate, as shown in Fig. 10. This is consistent with the increase in the temperature gradient near the droplet surface observed in Fig. 7(b) which, as noted, is similar in both the weak and strong limits. In the absence of an external electric field, $M \approx 1.8$, in agreement with eq. (26) as shown in Fig. 3. The burning rate increases monotonically with increasing the Ohmic heating parameter $\chi$. We recall that the aforementioned calculations assumed that the ratio $\chi / \eta=c=10$, a parameter that depends only on the combustion mixture. The results, however, remain quali-

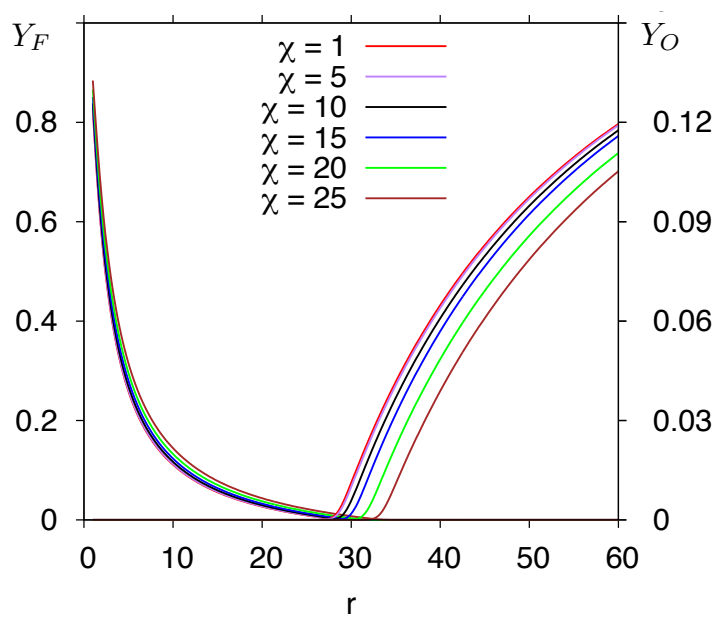

(a) $Y_{F}$ and $Y_{O}$ in the combustion field

Figure 8: Profiles of the mass fraction of the fuel and oxidizer for various values of the Ohmic heating parameter $\chi$.

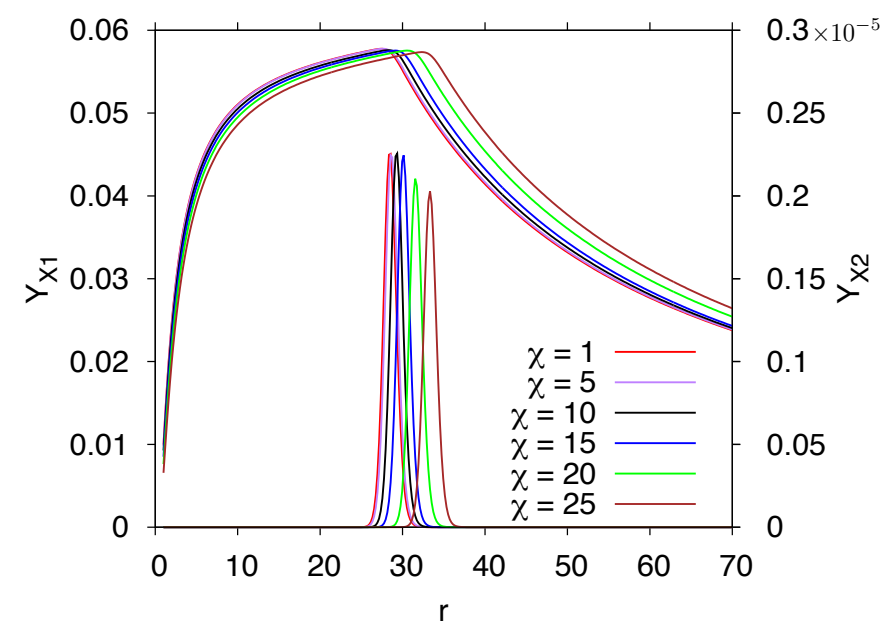

Figure 9: Profiles of the mass fraction of the radicals $X_{1}$ and $X_{2}$ for various values of the Ohmic heating parameter $\chi$. 
tatively the same for different fuel or oxidizer, or different values of $c$. An increase or decrease in $c$ merely changes the strengthening effect of the Ohmic heating term in the energy equation (22) through $\chi$. Curves for different values of $c$, with $0<\eta<2.5$, are shown in Fig. 11 .

\subsection{Flame Extinction}

The results presented above were for a specified Damköhler number $D a_{1}$, sufficiently large that corresponds to intense burning, as evident from Fig. 8 that shows that the fuel and oxidizer are completely consumed in the reaction zone. It is known that when reducing the Damköhler number, fuel and/or oxidizer leak through the reaction zone and the flame temperature drops. This leads eventually to flame extinction, occurring when the flame temperature is too low and there is no sufficient mixing of the reactants for the chemical reaction to proceed. The response curve showing the dependence of the flame temperature on the Damköhler number is typically S-shaped, with the upper part tracing burning states corresponding to complete combustion at large Damköhler numbers, down to extinction at the turning point. (The lower branch corresponds to unstable states that are physically unattainable). It is interesting to examine the influence of the imposed electric field on the extinction limit. As mentioned earlier, due to the dependence of Ohmic heating on $\nabla \phi$, the extinction point is independent of the limits of electric field. In Fig. 12 we show response curves corresponding to different values of the Ohmic heating parameter $\chi$. The results suggest that the influence of the electric field is in extending the flammability limit of the diffusion flame, as expected because the primary effect of the field in the

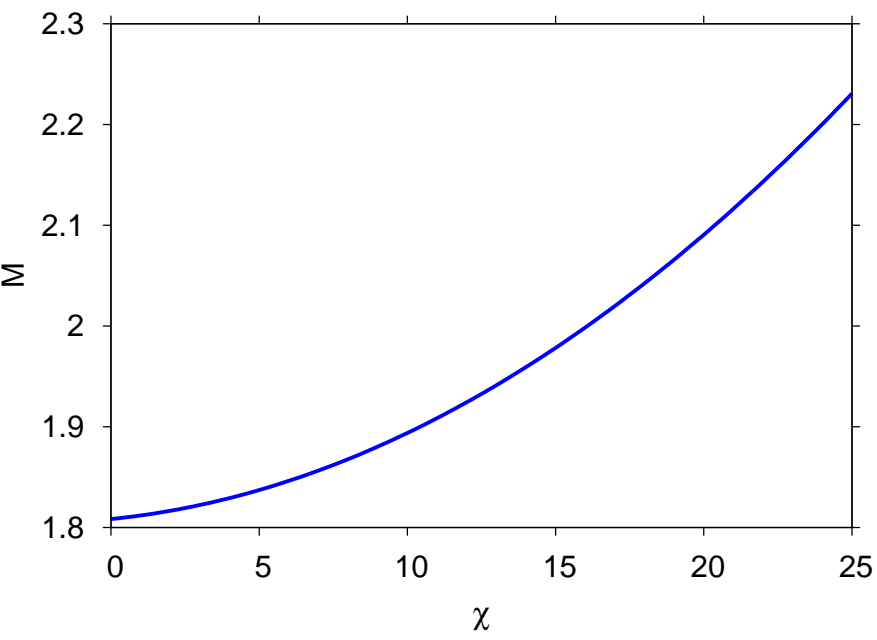

Figure 10: Variation of the mass burning rate $M$ as a function of the Ohmic heating parameter $\chi$.

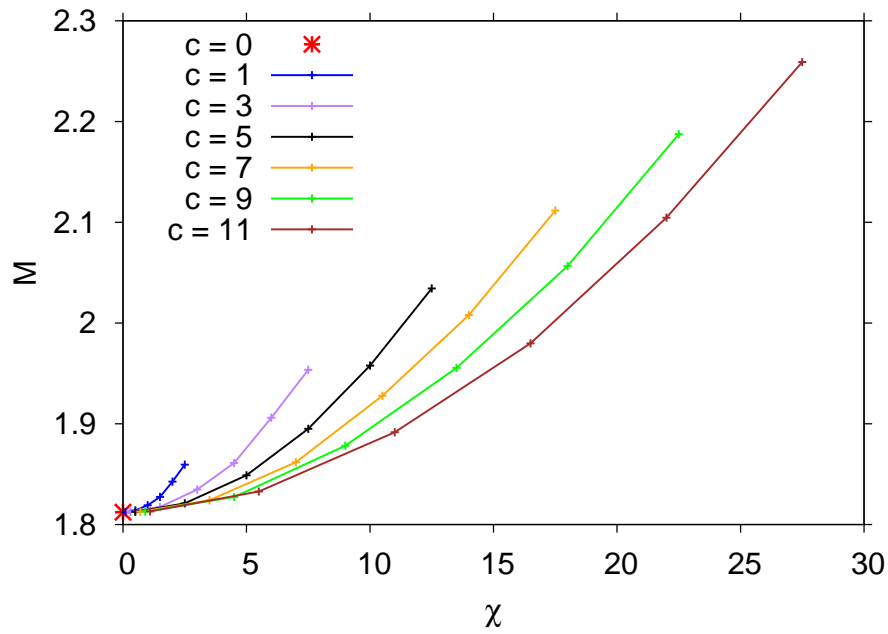

Figure 11: Variation of $M$ for different values of control parameter $c$, with $\eta$ varying in the range $[0,2.5]$.

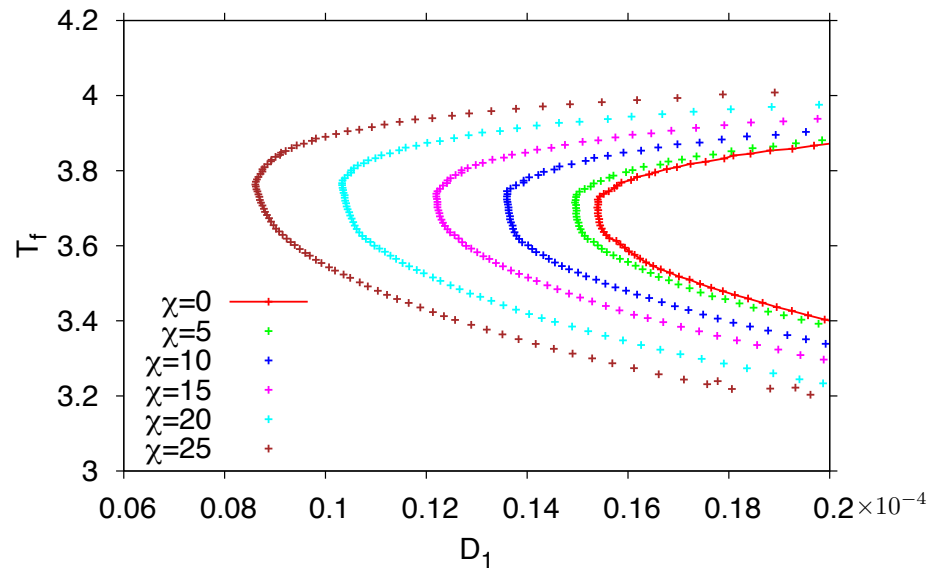

Figure 12: Response curve showing the dependence of the flame temperature $T_{f}$, defined as the maximum temperature in the domain, on the scaled Damköhler number $D_{1}$.

present configuration is to increase the mass burning rate which strengthens the burning process.

\subsection{Ionic Wind}

The focus so far has been on the influence of the electric field through the Ohmic heating appearing in the energy equation. An additional effect appearing in the momentum equation is the net body force acting on the charged species, known as the ionic wind. Due to the symmetry of the droplet configuration, the momentum equation uncouples from the remaining equations and serves only to determine the pressure field. In dimensionless form, the momentum equation (10) after ignoring the viscous forces takes the form

$$
\frac{d p}{d r}=-\frac{M}{r^{2}} \frac{d v}{d r}-\beta \rho\left(\frac{d \phi}{d r}\right)\left(Y_{p}-Y_{n}\right)
$$




$$
\beta=\operatorname{Pr}\left\{\frac{q \mathbb{V} \mathcal{N}_{A} \rho_{\infty} /\left(W_{p} r_{o}\right)}{\mu \mathcal{D}_{t h} / r_{o}^{3}}\right\} .
$$

is a measure of the force due to the electric field relative to the viscous force (per unit volume). In the absence of an external electric field, the pressure near the droplet falls off from its ambient value because of the Stefan flow, namely the flow of fuel vapor emanating from the droplet as a result of vaporization. The pressure is lowest near the droplet surface and increases outwards to its ambient value. When applying an electric field, the negative velocity gradient is balanced by the ionic wind, of magnitude proportional to the strength of the electric field, leading to a pressure rise near the droplet surface that decays slowly downstream to its ambient value. Shown in Fig. 13 is the pressure across the combustion field in the weak and strong field limits, and in the absence of an electric field. It is evident that the ionic wind is effective in reverting the pressure near the droplet surface even in the weak field limit. Within few droplet radii the pressure becomes nearly constant as in the pure vaporization case. In the strong field limit the ionic wind leads to a relatively large pressure rise near the droplet surface (nearly ten-folds) that decays slowly downstream. Recall that the variations in the dynamic pressure are typically a small fraction of the overall ambient pressure, consistent with the low Mach number assumption. The ionic wind has no direct effect on the flame in the present configuration, because the flame typically stands sufficiently far downstream, at a location where the electric field is already very weak. The sharp drop in electric field is due to

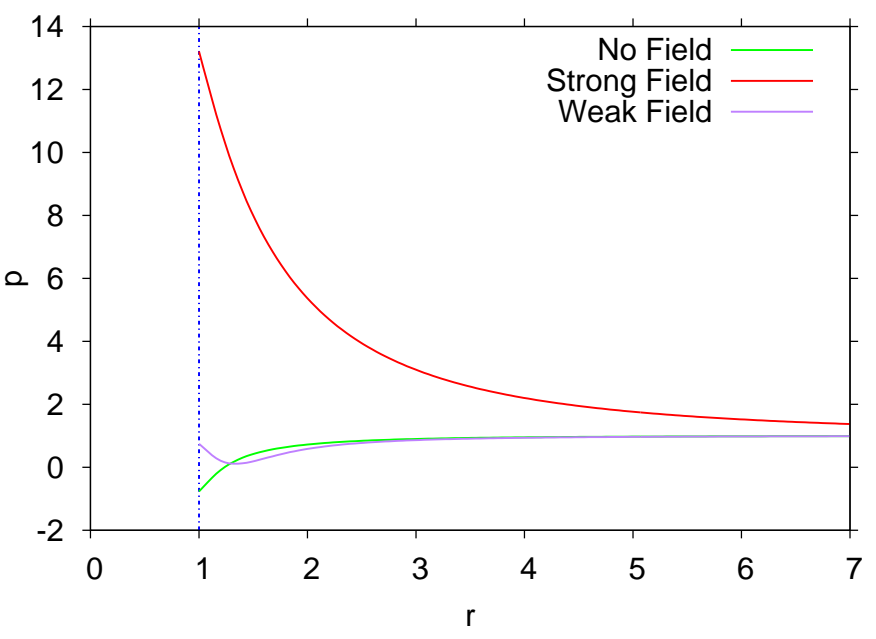

Figure 13: The pressure distribution across the combustion field in the strong and weak field limits, and in the absence of an external electric field. the hyperbolic-like decrease in electric potential, as seen in Fig. 4. Indeed, depending upon the electric field configuration, it is possible to envisage a situation where the ionic wind would affect the flame position.

\section{Conclusions}

We have systematically developed a framework to theoretically study the effects of an externally applied electric field on the characteristics of a diffusion flame. For the same, a three-step reduced chemical mechanism was introduced to mimic the production and destruction of charged species through chemi-ionization for general hydrocarbon fuels. The objective is to identify the mechanisms that affect flame behavior, such as flame structure, flame standoff distance, mass burning rate, and flame extinction conditions. Two main mechanisms are identified, namely kinetic and body force effects. Kinetic effects are highlighted by a drift velocity that modifies the net diffusive flux of the charged species increasing the Fickian diffusion velocity for positively charged species and decreasing it for negatively charged species. As a consequence, there is an appreciable difference in the mass fraction distribution of positive and negative ions that strongly depends on whether the internal electric field created by the charge distribution of the ionic species is sufficiently strong to affect the external electric field. The additional body force is highlighted via the "ionic wind" that affects the momentum balance and a Ohmic heating effect that could be positive or negative depending on the flow configuration and direction of the electric field. Due to the imposed symmetry, the ionic wind effect in the droplet problem acts only to modify the radial pressure gradient in the combustion field, with no implication on the flame. In more general circumstances this additional force could have an effect on the flame position and consequently on other flame characteristics. Ohmic heating on the other hand was observed to act as a heat source for the droplet setup, leading to a slight increase in flame temperature, enhancement in the burning rate, and extension of the flammability limits.

\section{Acknowledgments}

This work was partially supported by the U.S. Air Force Office of Scientific Research, 917 AFUOFMO 678C00043936-1, program manager Dr. M. Birkhan.

\section{References}

[1] S. Burke, T. Schumann, Industrial and Engineering Chemistry 20 (1928) 998. 
[2] A. Liñán, Acta Astronautica 2 (1974) 1009.

[3] S. Cheatham, M. Matalon, Journal of Fluid Mechanics 414 (2000) 105-144.

[4] M. Smooke, I. Puri, K. Seshadri, Proceedings of the Combustion Institute 21 (1988) 1783-1792.

[5] M. Smooke, P. Lin, J. Lam, M. Long, Proceedings of the Combustion Institute 23 (1991) 575-582.

[6] B. Lewis, Journal of the American Chemical Society 53 (1931) 1304-1313.

[7] H. F. Calcote, Combustion and Flame 1 (1957) 385-403.

[8] J. Goodings, D. Bohme, C. Ng, Combustion and Flame 36 (1979) 45-62.

[9] J. Goodings, D. Bohme, C. Ng, Combustion and Flame 36 (1979) $27-43$.

[10] H. Calcote, Symposium (International) on Combustion 8 (1961) 184-199.

[11] A. Eraslan, R. Brown, Combustion and Flame 74 (1988) 19-37.

[12] T. Pedersen, R. Brown, Combustion and Flame 94 (1993) 433448.

[13] J. Prager, U. Riedel, J. Warnatz, Proceedings of the Combustion Institute 31 (2007) 1129-1137.

[14] H. Jaggers, A. V. Engel, Combustion and Flame 16 (1971) 275285.

[15] Z.-G. Yuan, U. Hegde, G. M. Faeth, Combustion and Flame 124 (2001) 712-716.

[16] T. Ueda, O. Imamura, K. Okai, M. Tsue, Proceedings of the Combustion Institute 29 (2002) 2595-2601.

[17] K. Okai, T. Ueda, O. Imamura, M. Tsue, M. Kono, J. Sato, D. Dietrich, F. A. Williams, Combustion and Flame 136 (2004) 390393.

[18] K. Yamashita, O. Imamura, Combustion Science and Technology 180 (2008) 652-673.

[19] E. K. Anderson, J. A. Koch, D. C. Kyritsis, Combustion and Flame 154 (2008) 624-629.

[20] S. Won, S. Ryu, M. Kim, M. Cha, S. Chung, Combustion and Flame 152 (2008) 496-506.

[21] M. Kim, S. Chung, H. Kim, Proceedings of the Combustion Institute 33 (2011) 1137-1144.

[22] M. Papac, D. Dunn-Rankin, Combustion Theory and Modelling 12 (2007) 23-44.

[23] M. Sánchez-Sanz, D. C. Murphy, C. Fernandez-Pello, Proceedings of the Combustion Institute (2014).

[24] D. Bradley, The effects of electric fields on combustion processes, ACADEMIC PRESS, INC., pp. 331-400.

[25] R. Heinsohn, P. Becker, Combustion technology: Some modern developments.(A 74-44126 22-33) New York, Academic Press, Inc., 1974, (1974) 239-273.

[26] A. Fialkov, Progress in Energy and Combustion Science 23 (1997) 399-528.

[27] M. Belhi, P. Domingo, P. Vervisch, Combustion and Flame 157 (2010) 2286-2297.

[28] L. Landau, E. Lifshitz, Fluid Mechanics, Pergamon Press, 1958.

[29] J. Lawton, F. J. Weinberg, Electrical aspects of combustion, Clarendon Press, Oxford, 1969.

[30] J. Green, T. Sugden, Proceedings of the Combustion Institute 9 (1963) 607-621.

[31] N. Peters, Lecture Notes in Physics 241 (1985) 90-109.

[32] C. K. Law, Combustion Physics, Cambridge University Press, 2006. 